\title{
REVIEW
}

\section{Gene therapy in nonhuman primate models of human autoimmune disease}

\author{
BA $\mathrm{t}^{\prime} \mathrm{Hart}^{1,4}, \mathrm{M}$ Vervoordeldonk ${ }^{2}$, JL Heeney ${ }^{3}$ and PP Tak ${ }^{2}$ \\ ${ }^{1}$ Department of Immunobiology, BPRC, Rijswijk, The Netherlands; ${ }^{2}$ Division of Clinical Immunology and Rheumatology, Department of \\ Medicine, Academic Medical Centre, University of Amsterdam, The Netherlands; ${ }^{3}$ Department of Virology, BPRC, Rijswijk, The \\ Netherlands; and ${ }^{4}$ ErasMS Centre for MS Patient Care, Research and Expertise, Rotterdam, Rijswijk, The Netherlands
}

Before autoimmune diseases in humans can be treated with gene therapy, the safety and efficacy of the used vectors must be tested in valid experimental models. Monkeys, such as the rhesus macaque or the common marmoset, provide such models. This publication reviews the state of the art in monkey models for rheumatoid arthritis and multiple sclerosis and the (few) gene therapy experiments that have been performed in these models.

Gene Therapy (2003) 10, 890-901. doi:10.1038/sj.gt.3302017

Keywords: rhesus monkey; common marmoset; mutiple sclerosis; rheumatoid arthritis

\section{Introduction}

The impressive development in the biotechnology field has facilitated the generation of safer and more effective therapies for the treatment of autoimmune diseases in the human population. Examples of biotechnologyderived pharmaceuticals (biologicals) that have now reached the clinic include monoclonal antibodies or antagonist proteins for TNF- $\alpha$ (Infliximab, Etanercept) or interleukin-1 (IL-1) (Anakinra) for treatment of chronic inflammatory disorders like rheumatoid arthritis (RA) and inflammatory bowel disease ${ }^{1}$ or cytokines, such as interferon- $\beta$ for multiple sclerosis (MS). ${ }^{2}$ Many more targets and diseases are being investigated in a variety of experimental models. The main lesson from all these studies is that experimentally induced autoimmune diseases can be safely treated with biological molecules. However, the in vivo half-life of most biological agents is relatively short. The repeated administration of high systemic doses required to achieve therapeutic levels in affected organs may lead to deleterious side effects. Furthermore, the therapeutic window of biological molecules is often limited by the induction of antibodies capable of blocking the therapeutic itself. Targeted gene transfer provides the possibility to achieve therapeutic effects while circumventing the above-mentioned complications.

A critical factor in the successful development of somatic gene therapy is the availability of valid animal models in which the safety and efficacy of constructs designed for treatment of patients can be accurately evaluated. In many cases, biological molecules that are designed to work in humans are insufficiently active in rodents and cannot be tested. Nonhuman primates with

Correspondence: Dr BA t'Hart, Biomedical Primate Research Centre, PO Box 3306, 2280 GH Rijswijk, The Netherlands their greater immunological and biological similarity to humans are in great demand as models for preclinical evaluation of highly specific biological therapeutics. Several immunomodulatory therapies seem to work less effectively in primates than in inbred rodents. This may be because of the fact that the immune system of a few months old inbred rat or mouse is less mature than that of an adult primate or human. The value of nonhuman primate disease models is, therefore, that they predict which experimental therapies that work well in rodents could also be effective in clinical trials.

At the time we wrote this article, we found in a Medline search combining the keywords 'gene therapy' and 'primate' about 400 hits; while combination of 'gene therapy' and 'monkey models' gave around 80 hits and combination of 'gene therapy' and 'autoimmune disease' gave 323 hits. In contrast, a focused search on the keywords 'gene therapy' and 'autoimmune disease' and 'primates' yielded only four titles. More frequently addressed disorders are eye and lung disease, transplant rejection, and immunodeficiency. It can thus be concluded that there is considerable research activity on the application of gene therapy as treatment of autoimmune disease and that also considerable experience exists with gene therapy in nonhuman primates. However, despite the promising results, gene therapy has only rarely been tested in nonhuman primate models of human autoimmune disease. Nevertheless, such models could provide crucial information on the suitability of gene transfer vector systems in vivo before trials in humans are undertaken.

The purpose of this article is to discuss established nonhuman primate models of human autoimmune disease, focusing on arthritis and MS. We will review data from our own studies showing that these models are fit for use as preclinical test systems for gene therapies. 


\section{Nonhuman primates as model of human autoimmune disease}

The most widely used nonhuman primates in autoimmune disease research are Old World monkey species, in particular the rhesus and cynomolgus macaque (Macaca mulatta and M. fascicularis). More recently, disease models in New World monkeys have been established, such as common marmosets (Callithrix jacchus) or cottontop tamarins (Saguinus oedipus). The common ancestor of macaques and humans dates back to 35 million years, while New World monkeys are thought to have shared a common ancestor with humans 55 million years ago., ${ }^{3,4}$ This evolutionary proximity is reflected by a high degree of similarity at the level of polymorphic gene families, such as those encoding major histocompatibility complex or T-cell receptor molecules or immunoglobulins, and also of leukocyte surface molecules and immune regulatory molecules, such as cytokines and chemokines. Of importance for gene therapies that make use of virus-based constructs is the fact that both human and nonhuman primates can be infected with similar types of viruses and that infections often follow a similar course.

Certain autoimmune diseases are primarily caused by autoantibodies. While T cells do not seem to play a direct pathogenic role, they support autoantibody formation. Prevalent examples are autoimmune thyroiditis, myasthenia gravis (MG), systemic lupus erythematosus (SLE), and Guillain-Barré syndrome. Actively induced models of $\mathrm{MG}^{5}$ and $\mathrm{SLE}^{6}$ have been established. However, in view of the already mentioned high antigenic similarity of nonhuman primates and humans surprisingly few attempts have been made to induce such antibody-based autoimmune diseases in nonhuman primates by adoptive transfer of patient sera.

T-cell-dependent autoimmune diseases are much more widely investigated in nonhuman primates, RA and MS in particular. In this article, we will primarily focus on these two diseases in which the feasibility of gene therapy has been tested in the past several years.

\section{Nonhuman primate models of arthritis}

\section{Rhesus monkey model of collagen-induced arthritis}

RA is the most prevalent immune-mediated arthritic disease in the human population. The pathological hallmarks of RA are the hyperproliferation of synovial tissue (synovitis) and the progressive destruction of joint cartilage and bone. The hyperplastic synovium becomes infiltrated with blood-borne $\mathrm{T}$ cells and macrophages already at an early stage, before warmth and swelling of the joints are observed. ${ }^{7}$ The infiltrated $T$ cells are thought to contribute significantly to the pathophysiological cascade of reactions that gives rise to the disease and are, therefore, among the favored targets of immunotherapy.

Spontaneous cases of arthritis and osteoarthritis have been found in free-ranging rhesus monkeys at an incidence of around 20\%, but are rare in animals kept under laboratory conditions. For this reason, actively induced models have been developed that can be established when needed. By immunization with collagen type II (CII) extracted from bovine cartilage in complete Freund's adjuvant (CFA), a reproducible RAlike autoimmune polyarthritis could be induced in rhesus $^{8,9}$ and cynomolgus macaques. ${ }^{10}$ The time of disease onset and the pattern of clinical signs may vary, probably reflecting the outbred character of this species. Bacterial antigens that have proven to be arthritogenic in susceptible strains of rats and mice fail to produce arthritis in rhesus monkeys. ${ }^{9}$

Collagen-induced arthritis (CIA) is a useful experimental model of human RA, which is widely used to investigate how pathogenic autoimmune mechanisms evoke joint inflammation and is used for the preclinical testing of novel intervention strategies. ${ }^{11}$ The rhesus monkey model of CIA was originally designed as a preclinical model of human rheumatic disease for efficacy testing of biotechnology-derived pharmaceuticals, which by their species-specificity cannot be tested in rodents. The model shares several features of the joint pathology with human arthritic disease, and also shows some important differences. RA is a chronic progressive disease, which may affect multiple organs. Autoimmunity to CII is not consistently found in RA patients, but autoantibodies to self-IgG molecules (rheumatoid factors) are frequently detected. In contrast, CIA is a spontaneously remitting autoimmune disease that lacks rheumatoid factors, and inflammation is confined to the synovial joints and spinal column. Whereas in RA women are two to three times more often affected than men, a female prevalence has been reported in cynomolgus monkeys but was found to be absent in rhesus monkeys. ${ }^{10}$

A single immunization with bovine CII in CFA induces a heterogeneous pattern of joint inflammation in a random sample of rhesus monkeys from the outbred colony kept at the Biomedical Primate Research Centre in Rijswijk (The Netherlands). ${ }^{12}$ Between 10 and $20 \%$ of the monkeys develop fulminant polyarthritis necessitating euthanasia within a few days after disease onset. At the other side of the spectrum there are about $30 \%$ nonresponders, which remain completely asymptomatic even after several booster immunizations. In the largest group of monkeys, the disease follows a more or less chronic course of variable severity and duration, which wanes spontaneously. In its most typical presentation, arthritis starts with warm and swollen ankles, and/or wrists (Figure 1). At a later stage, inflammatory swelling in one or more of the interphalangeal, (meta)carpal, and (meta)tarsal joints is observed. Finally, arthritis develops in knees, hips, and elbows. Occurrence of spondylitis is only rarely observed. The large body of data from rodent models indicates that CIA induction depends on synergy of delayed-type hypersensitivity and immune complexmediated inflammatory mechanisms. ${ }^{13-15}$ This also seems to be the case in the rhesus monkey model of CIA.

A systematic comparison of responder versus nonresponder monkeys and therapeutic intervention studies revealed genetic and immunological factors associated with CIA susceptibility. The fact that our Indian origin rhesus monkey colony has been pedigreed on the basis of segregation of major histocompatibility complex (MHC) class I and II polymorphisms has facilitated the identification of the MHC class I serotype Mamu-A26 as a dominant resistance marker of CIA. In a large cohort of young adult monkeys, $95 \%$ of the CIA-resistant animals were Mamu-A26 positive, whereas the marker was absent 

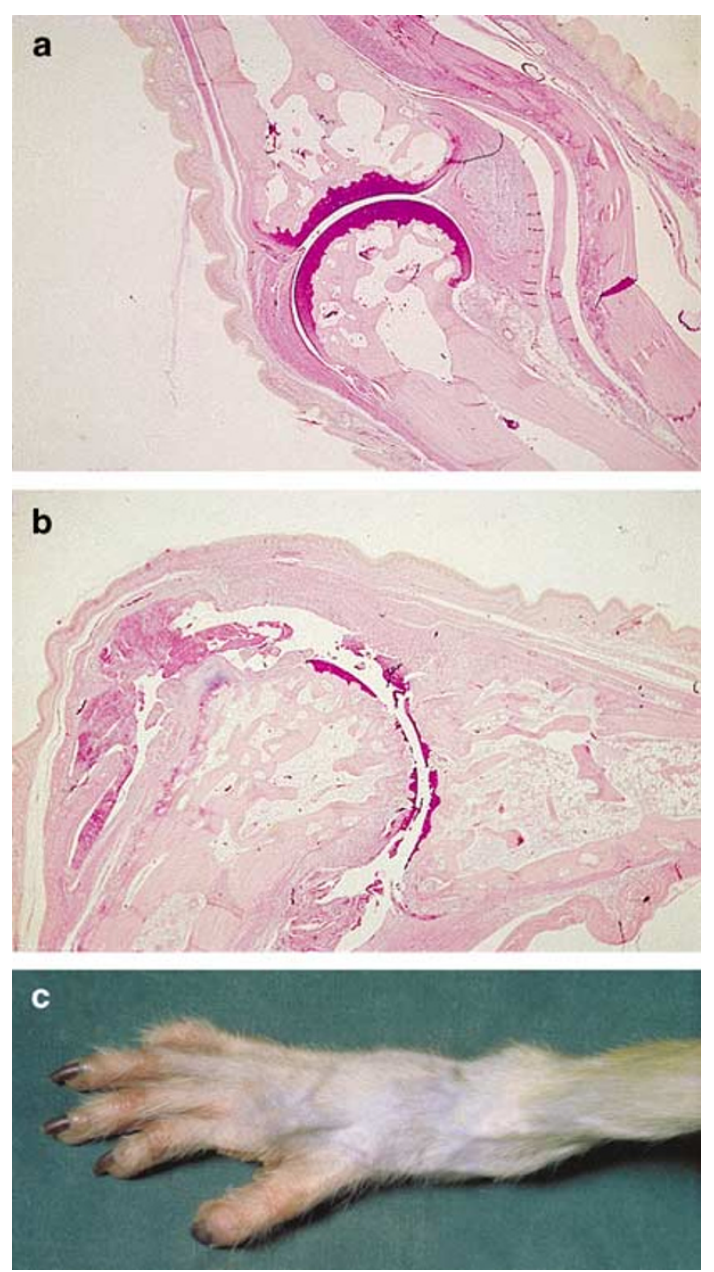

Figure 1 Macroscopic and microscopic aspects of the rhesus monkey CIA model. The bottom figure (c) shows a rhesus monkey hand with prominent inflammatory swelling of the interphalangeal joints and wrist. PAS staining of proximal interphalangeal finger joint from a healthy (a) and severely arthritic (b) rhesus monkeys shows the dramatic erosion of joint cartilage in this model.

in $99 \%$ of the CIA-susceptible monkeys. ${ }^{16}$ As no influence of $M a m u-A 26$ on susceptibility to autoimmune encephalomylitis exists, we hypothesized that the resistance mechanism is specific for the disease-inducing antigen. ${ }^{17}$ Molecular analysis revealed that the MamuA26 serotype may represent a set of Mamu-B alleles (unpublished observations). However, the mechanism of the Mamu-A26-associated resistance to CIA still remains an enigma. The only immunological feature of MamuA26-positive monkeys that could be correlated with disease resistance was their incapacity to produce antiCII IgM antibodies, while the absolute levels and epitope specificity of anti-CII IgG antibodies did not differ between Mamu-A26-positive and -negative monkeys. ${ }^{12,18}$ This could be relevant for disease induction, since previous work has clearly shown the important role of IgM antibodies in the initial autoimmune attack on the cartilage surface. ${ }^{19,20}$

The parallel analysis of CII-immunized Mamu-A26positive and -negative monkeys yielded a set of useful disease parameters that allow a more objective monitoring of the disease process. Predictably, the loss of bodyweight is a reliable indicator of disease onset and remission. Moreover, the two main pathological processes in CIA, synovial inflammation and joint destruction, can be measured independently using plasma Creactive protein levels and urinary excretion rates of collagen crosslinks, respectively. ${ }^{21}$

The beneficial effect of prophylactic T-cell-directed therapy with cyclosporin $\mathrm{A}^{22}$ or daclizumab, a humanized anti-CD25 antibody, ${ }^{23}$ suggests that $\mathrm{T}$ cells play an important role in the onset of arthritis. Consistent with this view, we observed infiltration by $\mathrm{T}$ cells in synovial biopsies obtained before the development of clinical signs of arthritis. ${ }^{7}$ However, it is difficult to prove the pathogenic role of synovial T cells with adoptive transfer, since suitable syngeneic recipients are lacking in outbred species.

\section{Towards gene therapy for RA}

The potential benefits of gene therapy for the treatment of RA have been discussed in several recent reviews. ${ }^{24-27}$ Preclinical studies have revealed that therapeutic genes can be efficiently delivered to the synovium using either intra-articular injection of certain viral vectors or local delivery of cells that were transduced ex vivo. In addition, the systemic delivery of genes by either ex vivo or in vivo gene therapy has therapeutic effects on the onset or progression of disease in different animal models of RA. ${ }^{28-30}$ Fibroblast-like synoviocytes (FLS) have become a major target for ex vivo gene therapy in RA. For systemic approaches also lymphocytes and, more recently, dendritic cells have been used successfully. ${ }^{31,32}$

In order to facilitate the uptake and expression of the gene of interest, several types of vectors are used. The most efficient vectors are modified/adapted naturally occurring viruses. The characteristics of the various viral vectors differ in terms of efficiency, cytotoxicity, antigenicity, size of exogeneous DNA they will accept, as well as their ability to infect nondividing cells and integrate. The most commonly used viral vectors are derived from retroviruses, adenoviruses, adeno-associated virus (AAV), and to a somewhat lesser extent herpes simplex virus (HSV). Replication-deficient adenoviruses, which have high transduction efficiency, are currently the most widely used in preclinical and clinical studies. The commonly used adenoviral vector for gene therapy is based on adenovirus type 5. However, most humans already carry antibodies against this type of adenovirus and there is evidence that a single administration of a therapeutic useful dose of adenovirus generates an immune response that is sufficiently strong to prevent successful readministration of the same vector. In addition, there was dose-dependent liver and skeletal muscle toxicity as well as coagulation abnormalities and a decrease in platelet counts after use of a firstgeneration adenoviral vector in rhesus macaques. ${ }^{33}$ Therefore, it is unlikely that these first-generation adenoviral vectors will be used in a clinical setting. Interestingly, helper-dependent ('gutless') adenoviral vectors, which do not induce an immune response and mediate prolonged transgene expression, are currently under development and these may be less toxic.

HSV-based vectors, which have been used in a rabbit model of arthritis, are able to transduce a wide variety of cells, but have inflammatory capacity because of the expression of viral proteins. Further development is 
necessary before administration into human joints can be studied. Another potentially interesting vector is AAV, since the parental wild-type AAV has never been shown to have any pathogenic effect. Recent advances in methods for generating large-scale, adenovirus-free preparations have brought wider interest in the use of this vector system, also for RA. AAV2 has been used in different animal models of RA for systemic ${ }^{34}$ and local gene therapy. ${ }^{35}$ A nonhuman primate study involving endotracheal delivery of AAV to specific segments of the macaque lung did not show any sign of inflammation induced by the vector. ${ }^{36}$ AAV vectors are currently being used in several clinical gene therapy trials, but AAV has as yet not been used in trials with RA patients. Few studies have investigated the potential of different serotypes of AAV, which may result in additional vectors capable of efficient gene transfer. The majority of retroviral vectors are based on the Moloney murine leukemia virus (MMLV). These vectors are usually applied for ex vivo gene therapy in clinical trials. Moreover, this vector has been used in the first gene therapy clinical trial in RA patients (see below). Studies with nonviral vectors have also been reported, but therapeutic efficiency has as yet not been demonstrated in RA models.

The number of possible therapeutic genes that may be used for gene therapy of arthritis is still growing. Blockade of the effects of IL- 1 and TNF- $\alpha$ by gene therapy has shown prevention of disease progression and joint destruction in several animal models of arthritis. ${ }^{35,37-39}$ The combination of TNF blockade and IL-1 blockade by adenoviral gene therapy in arthritic rabbits was shown to be even more effective than blockade of either cytokine alone. ${ }^{39}$ The transcription factor nuclear factor $\kappa \mathrm{B}(\mathrm{NF}-\kappa \mathrm{B})$, which induces activation of many proinflammatory genes including TNF and IL-1, may be a potential target for local gene therapy. However, its widespread role could cause toxicity if used systemically. Blocking NF- $\kappa \mathrm{B}$ activity by direct intra-articular gene therapy with an adenoviral vector containing a dominantnegative IкB kinase $\beta$ (IKK) mutant clearly ameliorated rat adjuvant arthritis. ${ }^{40}$ Other strategies include the transfer of genes encoding anti-inflammatory cytokines, which may suppress and counterbalance Th1-driven responses and inhibit pro-inflammatory cytokine production. Examples include IFN- $\beta,{ }^{30}$ IL- $4,{ }^{41}$ IL-10, ${ }^{37}$ and IL-13, ${ }^{42}$ which have all been used successfully in animal models of RA.

Several factors may account for the increase in cellularity of rheumatoid synovial tissue, including inadequate apoptosis. Therefore, gene therapy might be used to induce apoptosis in the inflamed joints. Since the death factor Fas and its ligand FasL play pivotal roles in the induction of apoptosis of immune cells, adenovirusmediated FasL gene transfer was examined in arthritic joints and was shown to reduce inflammation and ameliorate murine CIA. ${ }^{43}$ Another way to induce apoptosis in synoviocytes would be by delivering the herpes thymidine kinase (TK) gene followed by administration of ganciclovir. This approach was used in rhesus monkeys with $\mathrm{CIA}^{44}$ and will be discussed below in more detail.

Angiogenesis, the formation of new blood vessels, is one of the earliest histopathologic findings in RA and appears to be necessary for pannus development. The efficacy of local expression of an antiangiogenic gene by ex vivo gene therapy using a retroviral vector was investigated in the murine CIA model. ${ }^{45}$ Local production of angiostatin in the knee was able to prevent the onset of CIA not only in the knee injected with genetically engineered cells, but also in uninjected ipsilateral joints. The injection of an endostatin-expressing lentiviral vector directly into the joints of human TNF-transgenic mice resulted in reduced vessel density in the synovial tissue associated with a decrease in the overall mean arthritis index. ${ }^{46}$

Two phase I studies have been performed in RA patients, one in the US and one in Germany. ${ }^{47,48}$ In these studies, a gene encoding human IL-1 receptor antagonist (IL-1RA) was transferred into the metacarpophalangeal (MCP) joints of female RA patients. Autologous synoviocytes were transduced ex vivo with a retroviral vector containing IL-1RA and the transduced cells were injected back into the MCP joints. All nine patients included in the US trial have completed the protocol, and the preliminary data confirmed that it is possible to transfer genes safely to human, rheumatoid joints and to express those genes intra-articularly. This was not only the first application of human gene therapy in arthritis patients, but also the first use of gene therapy approved for nonlethal disease. Based on these results a phase II trial has been planned.

\section{Synovectomy by adenovirus-encoded thymidine kinase and ganciclovir in CIA in rhesus monkeys}

To date the only published gene therapy study in a nonhuman primate model of arthritis was on removal of the hyperplastic synovium in CIA-affected rhesus monkeys. ${ }^{44}$ Although this study addressed a specific question, it also covered important basic aspects of gene therapy in RA, including the feasibility of in vivo gene transfer to tissue cells, the biodistribution of the vector, and finally the overall safety of the procedure. The experimental strategy in the gene therapy efficacy trial was to transduce specifically synovial cells in vivo using intra-articular injection of replication-defective adenovirus harboring the herpes simplex virus TK gene (Ad.TK). TK gene therapy made the synovial cells sensitive to intravenously administered ganciclovir, resulting in apoptotic cell death. On the basis of experiments with reporter genes (LacZ and luciferase), we concluded that the intra-articularly injected adenovirus only infected superficial intimal macrophages and FLS without penetration into the underlying tissue layers (Figure 2). Interestingly, although several of the monkeys had detectable serum titers of antiadenovirus antibodies, this did not appear to affect transgene expression nor was synovial inflammation enhanced. In light of the safety concerns one might have, it is important to note that there was no vector spill outside the injected joints.

A second study from the same group addressed the influence of the chosen promoter on transgene expression in the tissues. It was shown that the expression of reporter transgenes (LacZ/luciferase) in human and rhesus monkey synoviocytes is several fold higher when driven by a cytomegalovirus promoter than by an adenovirus major late promoter. ${ }^{49}$ 


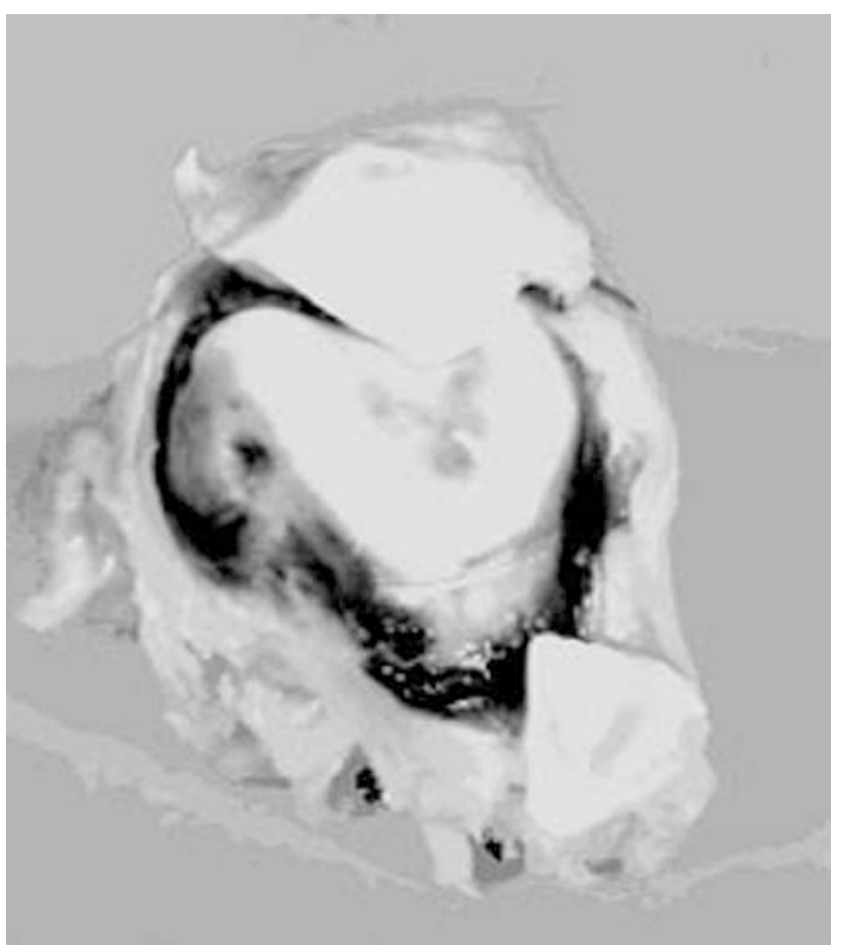

Figure 2 Transgene expression in an arthritic knee joint. Anatomical view of an arthritic knee joint from a rhesus monkey affected by CIA 3 days after injection of $10^{9} \mathrm{IU}$ AdLacZ that was stained for $\beta$-Gal. The blue area indicates the presence of the protein product of the lac $Z$ gene.

\section{Nonhuman primate models of MS}

\section{Monkey models of MS}

MS is a chronic inflammatory disease of the central nervous system (CNS). The pathological hallmark of MS and the most likely cause of the progressive neurological deficit is the CNS white matter lesion. Lesions are focal areas of inflammatory demyelination with a variable degree of axonal damage and gliotic scar formation. Although the etiologic trigger of MS is not known, most experts believe that autoreactive T- and B cells with specificity for components of CNS myelin contribute significantly to the disease. Similar to RA, MS is a clinically and pathologically heterogeneous disease. In a recently published broad survey of MS pathology at least four major types of lesions could be distinguished, which probably also represent four different disease mechanisms. ${ }^{50}$ The authors found evidence for an autoimmunebased cause in only two of the subtypes, namely type I formed by cellular autoimmune reactions and type II by synergy of cellular and humoral autoimmune mechanisms.

The two established nonhuman primate models of experimental autoimmune encephalomyelitis (EAE), in the rhesus monkey and the common marmoset, each resemble a subtype of MS. Acute EAE in rhesus monkeys resembles postinfectious forms of acute MS with based type I lesions, whereas the chronic EAE model in marmosets rather resembles the more common chronic form of MS. This remarkable species difference stays intact when EAE is induced in both species with the same protocol, including complete adjuvant emulsions
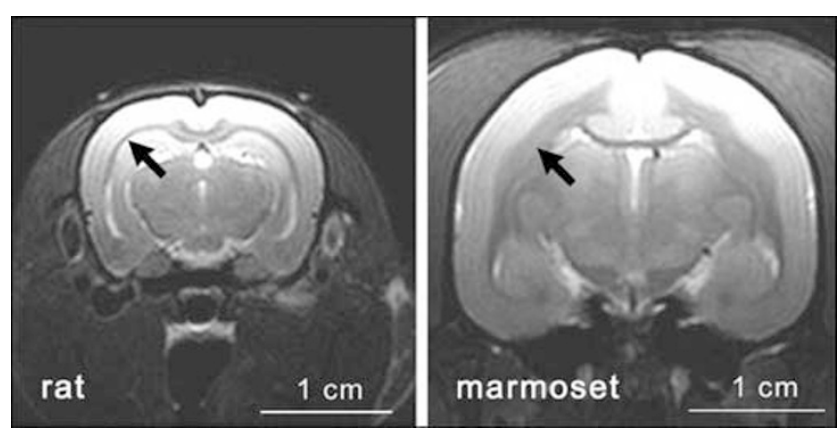

Figure $3 T_{2}$-weighted brain MR-images of a rat and a common marmoset. In $T_{2}$-weighted brain MR-images of naive animals, the white matter of a rat and a common marmoset appear as dark gray areas (arrow-head). The picture illustrates that the common marmoset brain contains much more white matter than the rat brain.

with human myelin or recombinant human myelin/ oligodendrocyte glycoprotein (rhMOG). ${ }^{51}$

A particularly useful aspect of both monkey models of MS is the fact that the ratio of white versus gray matter is much higher than in rodent models (Figure 3). Hence, magnetic resonance imaging (MRI) can be used to examine the development and spatial distribution of brain white matter in intact animals at different time points during the EAE course, comparable to its use in MS patients (Figure 4).

\section{Common marmoset model of experimental autoimmune encephalomyelitis}

Immunization with MS patient brain myelin emulsified in complete adjuvant induced chronic EAE in $100 \%$ of common marmosets in random samples from outbred colonies. ${ }^{52,53}$ The disease course in myelin-immunized marmosets varied among individual monkeys; cases with acute, primary progressive or relapsing-remitting/ secondary progressive disease were found. Pathologically this model is characterized by a heterogeneous pattern of lesions in the white matter of the brain and spinal cord. In a single monkey MS-like early active, late active, chronic inactive, and remyelinating lesions can be found at the same time. ${ }^{54}$ The pathomorphological aspect of the MS-type II-like lesions indicates that they are formed by specific inflammatory demyelination with sparing of axons.

It has become increasingly clear that MOG-reactive Tand $\mathrm{B}$ cells play a central role in the immunopathogenesis of this model. The $100 \%$ disease incidence in the outbred colony at the BPRC could be explained by the presence of a monomorphic MHC-DR molecule (Caja$\mathrm{DRB}^{*}$ W1201) in each tested monkey, which functions as a major restriction element for activation of MOG24-36 specific CD4+ T cells. ${ }^{55,56}$ Disease susceptibility was similar when EAE was induced with rhMOG or the encephalitogenic MOG peptide (14-36) that encompasses the specific CD4 cell epitope. Essentially, the same results were obtained by Genain et al. ${ }^{57}$ The beneficial effect of the phosphodiesterase inhibitor rolipram on myelininduced EAE supports the view that the pathogenic CD4 cells are of the Th1 type. ${ }^{57}$ While autoreactive T cells were sufficient for the initiation of CNS inflammation, specific demyelination was dependent on the presence of anti- 

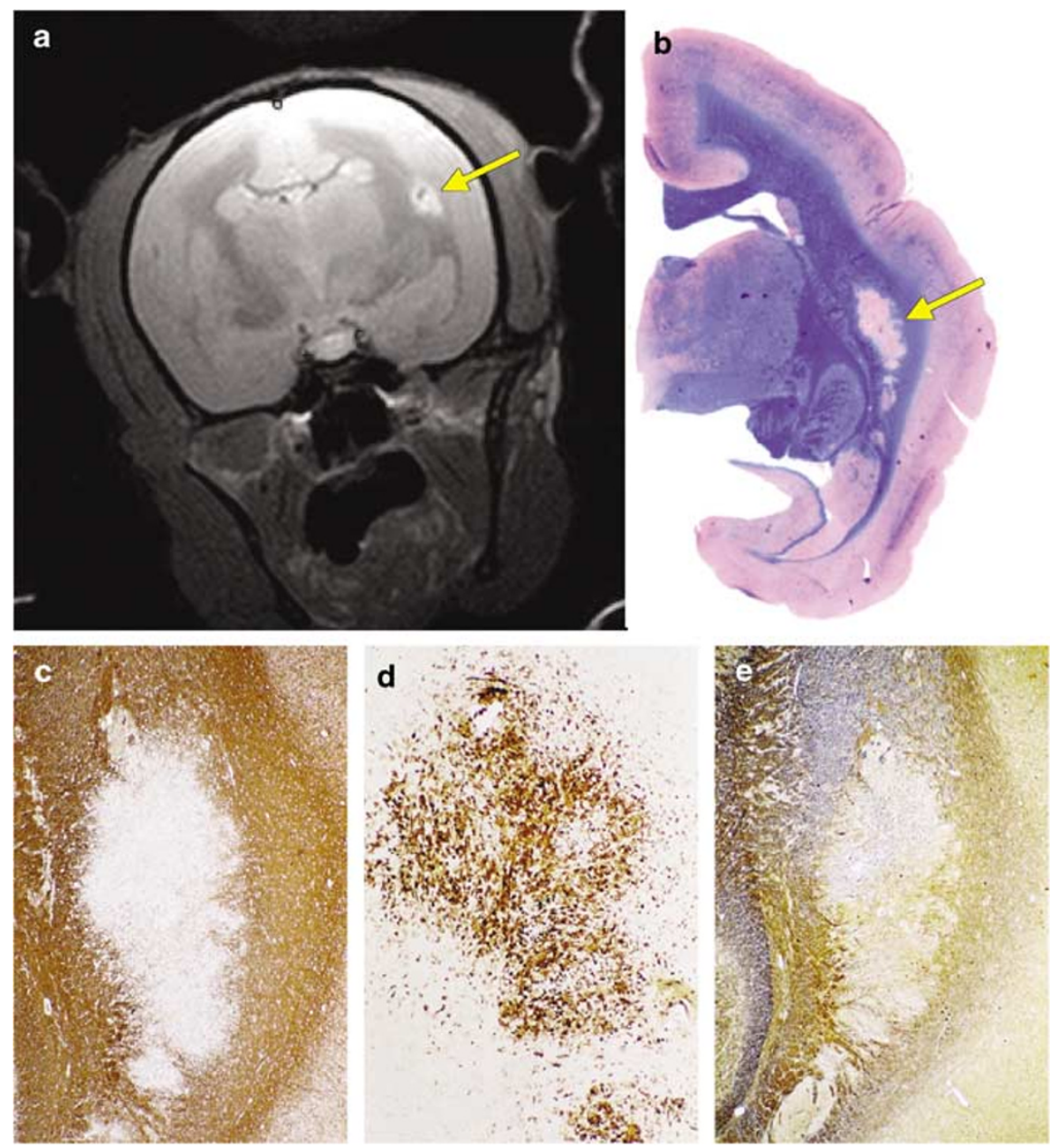

Figure 4 MS-like brain white matter lesion in marmosets. $T_{2}$-weighted brain $M R$-image was made of a common marmoset immunized with human myelin during clinical EAE. The MR-image (a) reveals a hyperintense region (arrow) in the right hemisphere. The histological staining (b; Klüver Barrera) shows that this region (arrow) is a demyelinated lesion. A magnification of the lesion in the bottom panel shows the extent of myelin loss (c; myelin staining for $2^{\prime}, 3^{\prime}$-cyclic nucleotide $3^{\prime}$-phosphodiesterase (CNP-ase)) of macrophage infiltration (d; MRP-14 antibody staining of infiltrating activated macrophages) and of axonal pathology (e; Bielschowski silver impregnation).

MOG antibodies, in particular those binding to conformational epitopes present in the extracellullar domain of the MOG molecule. ${ }^{58-60}$ This might explain the observation that expression of clinical EAE in monkeys immunized with a chimeric protein of human proteolipid protein (PLP) and myelin basic protein MBP is associated with spreading of the autoimmune reaction to MOG. ${ }^{60}$

The dominant role of MOG in the etiopathogenesis of marmoset EAE has prompted us to investigate in further detail the autoimmune mechanisms operating in the course of MOG-induced EAE. Marmosets immunized with rhMOG, which is a recombinant protein representing the 125 amino-acids long $\mathrm{N}$-terminal extracellular domain of human MOG, developed in all cases severe demyelinating EAE. Most monkeys displayed primary progressive EAE after an asymptomatic period of variable length. Monkeys with a relapsing-remitting disease course were only rarely found. As in MS there was marked discrepancy between the lesion load and the severity of neurological deficit. Using serial MRI, the presence of many large confluent brain white matter lesions could be demonstrated weeks before clinical signs developed. ${ }^{61}$

Therapeutic intervention studies shed some light on the immunopathogenic mechanisms underlying lesion formation and neurological deficit. It was shown that treatment with an antagonist mouse antibody to human CD40 (5D12) protected myelin-immunized marmosets against clinical EAE. However, clinical signs emerged as soon as antibody treatment was discontinued or when a neutralizing immune response developed. ${ }^{62}$ The mechanism of action of anti-CD40 therapy was investigated in the rhMOG-induced EAE model. ${ }^{63}$ Early administration of humanized 5D12 protected monkeys against the development of clinical EAE, but did not completely suppress inflammatory demyelination of the CNS. The proliferative response of axillary and inguinal lymph node cells towards MOG was at the same level in antibody-treated and placebo-treated monkeys. However, there was marked suppression of the anti-MOG autoantibody response.

IL-12, by which CD40 exerts in part its effects, is another interesting therapeutic target. ${ }^{64,65}$ This cytokine 
promotes Th1 responses to autoantigens, and is clearly expressed in the EAE-affected marmoset brain in early lesions. ${ }^{66}$ Antibody-mediated neutralization of IL-12 had a strong protective effect on both the clinical and neuropathological expressions of EAE in marmosets. ${ }^{67}$ Of special note in both studies was the observation that molecules with the size of an antibody could penetrate lesions and modify local immune responses.

\section{Rhesus monkey model of experimental autoimmune encephalomyelitis}

In its characteristic clinical course and destructive pathomorphological presentation of brain white matter lesions, the rhesus monkey model of EAE resembles acute forms of MS, such as disseminated encephalomyelitis and acute hemorrhagic encephalomyelitis. Recent unpublished data indicate that the MS type I-like lesions in this model are formed by a complex pathogenic process involving at least the synergy of anti-self and antiviral immune reactions. ${ }^{68}$ The influence of MHC class II genes on disease susceptibility, ${ }^{17}$ the suppressive effect of anti-HLA-DR and anti-CD4 antibodies, ${ }^{69}$ and the induction of brain white matter inflammation with CSF pleiocytosis by autologous transfer of myelin-reactive Thelper 1 cell lines ${ }^{70}$ all support a central role of autoreactive Th1 cells in the EAE pathogenesis. Autoantibodies do not seem to play a direct pathogenic role in this model. ${ }^{68}$ This concept provided the basis for a gene therapy trial aimed at intra-CSF delivery of IL-4, a potent inhibitor of Th1 cell functions. This experiment will be discussed below.

\section{Modulation of experimental autoimmune encephalomyelitis by gene therapy}

The pathogenesis of autoimmune encephalomyelitis in primates involves cognate interaction between $\mathrm{T}$ cells and APC at multiple levels, namely in peripheral lymphoid organs where autoreactive $\mathrm{T}$ cells are primed, at the blood-brain barrier (BBB), within the brain parenchyma, and within the cervical lymph nodes and spleen where macrophages containing phagocytosed myelin drain to. ${ }^{71,72}$ Proinflammatory cytokines are formed as a result of T cell/APC interaction and play a central role in the orchestration of the immunological mechanisms that drive the pathogenesis of MS and are, therefore, considered important targets for immunotherapy. Systemic administration of neutralizing reagents (including antibodies and soluble receptors) and counter-regulatory cytokines has proven efficacy in rodent EAE models. However, several anticytokine therapies that worked well in EAE in mice and rats were less effective or even had detrimental effects in patients. This might be explained as owing to the timing of the treatment, the route of drug administration, and the fundamental immunological differences between rodents and primates.

Biological molecules that are considered to act within the brain and spinal cord have to take several hurdles on their way to the affected target organ. First, the molecule has to pass the $\mathrm{BBB}$, which under nonpathological conditions separates the brain from the peripheral circulation. When CNS inflammation occurs, the permeability of the BBB increases under the influence of inflammatory mediators, such as TNF- $\alpha$, IL-1 $\beta$, and matrix metalloproteinases. Under these conditions, large biological molecules, such as antibodies, could extravasate into the lesions ${ }^{62,67}$ (Figure 5). On the other hand, a significant proportion of active demyelinating lesions in the EAE-affected common marmoset brain did not show contrast enhancement at T1-weighted MRI, suggesting that the $\mathrm{BBB}$ is impermeable in these lesions. ${ }^{54}$ This might explain why therapeutic antibodies, such as antihuman CD40 or anti-human IL-12, are only partially effective when administered in a therapeutic fashion. ${ }^{62}$ Second, there is poor diffusion of larger (biological) molecules within the CNS white matter. The diffusion coefficient of a $20 \mathrm{kDa}$ protein in CNS tissue is in the order of $10^{-6} \mathrm{~cm}^{2} / \mathrm{s}^{73}$ This implies that it takes more than 3 days for a molecule of that size to diffuse $1 \mathrm{~mm}$ into the CNS tissue.

These hurdles could theoretically be circumvented by the use of gene therapy. Delivery of Th2 'protective' cytokine genes that are able to counteract the detrimental activity of Th1 cytokines has been studied in various EAE models. Both viral vectors and plasmids containing cytokine genes have been delivered systemically, either in the blood stream or using circulating encephalitogenic $\mathrm{T}$ cells. An alternative approach is direct administration of the construct into the CNS. By transferring cytokines
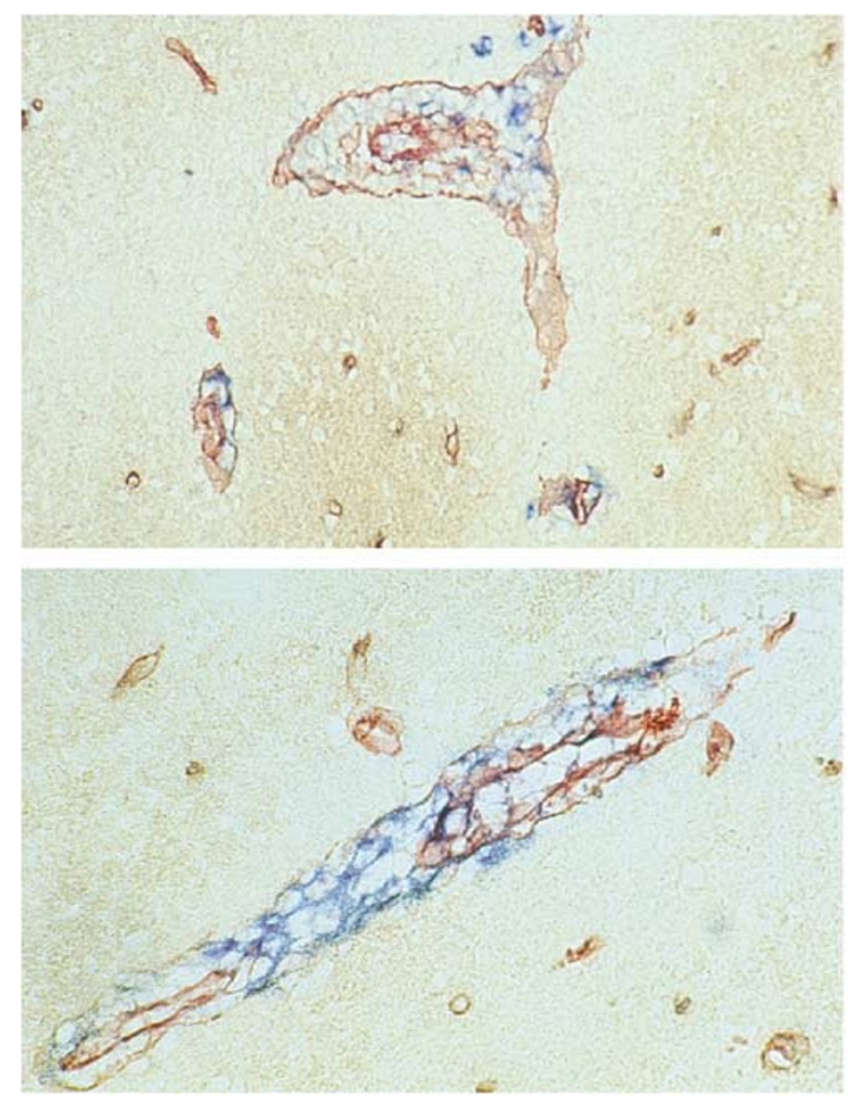

Figure 5 Localization of intravenously injected anti-CD40 antibody (blue) within the CNS. We injected $1 \mathrm{mg}$ of the mouse-anti-human CD40 antibody 5D12 in $0.5 \mathrm{ml}$ phosphate-buffered saline (PBS) into the vena saphena of an EAE-affected common marmoset $1 \mathrm{~h}$ before the monkey was killed. Cryosections of lesions were double-stained with anti-laminin antibody to demarcate the Virchows-Robin space (red) and with anti-mouse $I g G$ antibody to visualize localization of injected anti-CD40 antibody. The pictures show prominent localization of anti-CD40 within the perivascular space, but also within the surrounding white matter. 
in encephalitogenic $\mathrm{T}$ cells, 'suppressor' $\mathrm{T}$ cells are generated that will home to the sites of inflammation and demyelination in the CNS. After crossing the BBB, these cells could be reactivated to produce Th1-antagonizing molecules in situ, thereby inhibiting neighboring Th1 encephalitogenic T cells. Previous work has shown that IL-4 gene therapy ameliorated murine EAE, whereas TNF- $\alpha$ exacerbated the disease, and IL-10 was ineffective. ${ }^{74,75}$ An MBP-specific Th1 clone transduced with a retroviral vector containing the TGF $\beta$ gene delayed and ameliorated EAE development in mice when administered systemically before or at EAE onset. ${ }^{76} \mathrm{MBP}$-specific CD4+ T cells transduced with the IL-12 p40 gene were also able to reduce disease activity in EAE. ${ }^{77}$ Transduced CD4+ T cells were able to traffic into the CNS, as shown by the detection of bioluminescent lymphocytes, and exhibited long-term transgene expression. MBP-specific $T$ cells were also successfully used in EAE in Lewis rats, where a gene encoding nerve growth factor was shown to suppress the induction of EAE. ${ }^{78}$

The systemic injection of viral vectors has been reported in only one study. ${ }^{79}$ Viral vectors derived from Vaccinia virus were injected intravenously into mice affected by EAE. Vectors containing the genes encoding IL-6, TNF- $\alpha$, IL-1, IL-2, and IL-10, but not IL-4 or IFN- $\gamma$ ameliorated the disease. The data are limited, however, since clinical parameters were evaluated only during a 1week period and no histopathological evidence for a disease-protective effect was provided.

Plasmid constructs engineered with cytokine genes, which were injected intramuscularly or intracerebrally, have also been used in mice with EAE. Intracerebral administration of naked cytokine DNAs was not successful in the treatment of EAE. However, intramuscular injection of naked DNA coding for TGF $\beta$ or IL-4IgG chimeric protein into mice affected by MBP-induced EAE did reduce clinical and histopathological signs and symptoms. ${ }^{80}$ In these protocols, a pronounced downregulation of proliferating activity of MBP-specific T cells was observed. In contrast, cDNA coding for IL-4, TGF $\beta$, IFN- $\beta$, p75TNF-, and p55TNF receptor-Ig complexed with lipofectin-ameliorated EAE when injected intracerebrally 3 days before disease onset. ${ }^{81}$

Different therapeutic outcomes in EAE dependent upon the mode of delivery of IL-10 were found. IL-10 encoding DNA-liposome complexes were ineffective, and an adenoviral vector encoding murine IL-10 failed to inhibit EAE when injected into the parenchyma of the CNS, despite producing high levels of IL-10 protein. In contrast, intracerebral injection of fibroblasts transduced with a retroviral vector expressing IL-10 inhibited murine EAE. ${ }^{82}$ Overall, the results pointed to a limited effect of direct injection of genes encoding cytokines into the parenchyma of the CNS. Interestingly, an IL-4 encoding HSV-vector injected intrathecally did not only have a beneficial effect in murine EAE when administrated before the development of clinical signs, but also in established disease. ${ }^{83,84}$ The same approach was therefore evaluated in nonhuman primates with EAE .

\section{IL-4 gene therapy in rhesus monkeys with experimental autoimmune encephalomyelitis}

By its close proximity to chronic MS, the common marmoset model of EAE provides in general a very useful model to test new therapies for chronic MS. However, vector administration in a clinically relevant manner, for instance via the occipital or lumbar route, is hampered by the small size of the common marmoset. For this reason, we investigated in vivo gene therapy in myelin-induced acute EAE in rhesus monkeys using an intrathecally administered gene construct. ${ }^{85} \mathrm{HSV}$ deleted of genes encoding infected cell proteins (ICP) was used, because previous work has shown that this provides a safe and effective vector for gene transfer into the CNS in nonhuman primates. ${ }^{86}$ These vectors can accommodate multiple foreign genes, can be grown at high titer and, importantly, infect postmitotic cells including most resident cells within the CNS. The construct was a replication-deficient HSV type I encoding human IL-4 (abbreviated as TH4). In controls, the IL-4 gene was replaced by LacZ (THZ). The vectors were directly injected into the CSF, either into the cisterna magna or via lumbar puncture to mimic a possible future administration route in humans.

Tests with the THZ vector in naïve rhesus monkeys, killed 3 days after the vector injection, revealed widespread distribution of the virus in all ventricular and meningeal spaces, in association with limited penetration. Only the superfical cell layers directly facing the CSF were infected, namely ependymal, choroidal, and leptomeningeal cells. We did not observe signs of direct toxicity, apart from a transient and nonspecific inflammatory reaction because of the vector injection. IL-4 levels of 0.1 and $0.4 \mathrm{pg} / \mathrm{ml} \mathrm{CSF}$, produced by vectorinfected choroidal and ependymal cells, were measured in CSF collected 30 days after intrathecal injection of the TH4 construct in two naïve monkeys. Interestingly there was no vector leakage into peripheral blood, induction of anti-HSV-1 antibodies, or skewing of peripheral cytokine production.

Having demonstrated that vector administration had no overt negative side effects, we subsequently tested the potentially beneficial effect of TH4 on EAE. In all, 10 monkeys were randomly divided into two groups, immunized with human myelin in CFA, and followed up for 45 days. Four monkeys received $10^{8} \mathrm{PFU}$ of the control construct THZ 11 days after immunization; these animals developed acute clinical EAE with severe inflammatory brain lesions, while the fifth one developed milder signs of encephalitis. In contrast of the five TH4-treated monkeys, three remained completely devoid of any neurological and pathological signs (Figure 6). The beneficial effect of the TH4 gene therapy treatment was reflected by a clear reduction in mRNA expression for mRNA levels in CSF cells of TNF- $\alpha$ and MCP-1 in CSF cells. ${ }^{85}$

Taken together, these data indicate that intrathecal injection of an engineered herpetic vector is a safe and nontoxic approach for sustained delivery of IL-4 into the CSF. Moreover, treatment with the IL-4 encoding vector may clearly suppress clinicopathological signs of acute EAE in myelin-immunized monkeys.

\section{Future developments}

Selective targeting of specific molecules in affected organs by gene therapy remains a promising approach, which can be tested in nonhuman primate models of 


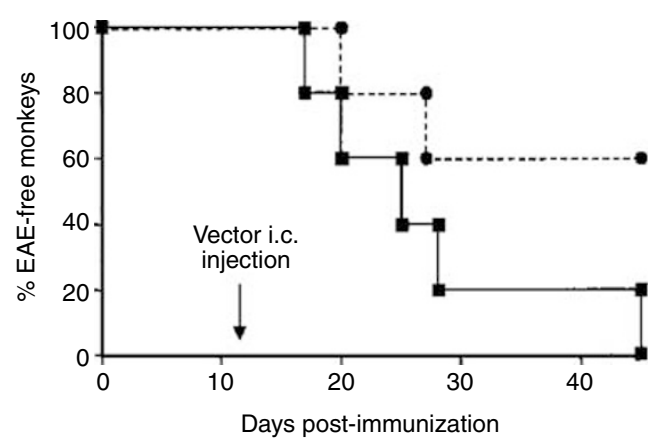

Figure 6 Gene therapy in EAE-affected rhesus monkeys. In all, 10 randomly selected monkeys were immunized with human myelin in complete adjuvant to induce autoimmune encephalomyelitis. At day 11 after immunization, we injected into the CSF of five monkeys HSV-1 vector containing the human IL-4 gene (circles) and in the remaining five monkeys HSV-1 vector containing the lacZ gene (squares) (copied with permission from Poliani et $\mathrm{al}^{73}$ ).

human disease. The site-specific delivery of 'regulatory proteins' that could correctly modulate the immune response holds promise as an innovative therapeutic strategy for immune-mediated diseases, including RA and MS.

Autoreactive T cells infiltrating the CNS white matter during the course of MS as well as antigen-specific T cells migrating into the inflamed joints in RA could represent attractive vehicles for therapeutic genes. Intriguing results have already been obtained in inbred rodent models of arthritis, ${ }^{87}$ neuritis, ${ }^{88}$ and encephalomyelitis. ${ }^{78}$ However, it is still an open question whether this approach will be feasible in the genetically heterogeneous human population. It is not clear, for example, if each patient will need autologous T cells as vector or that perhaps a limited set of donor cells could be sufficient. This research question can be well investigated in an outbred population of nonhuman primates.

A second issue that may be addressed in nonhuman primate models is the species specificity of vectors. Stable transduction of haemopoietic stem cells resulting in lineage-specific expression of therapeutic genes is one of the goals in gene therapy for autoimmune diseases. However, high-level transduction of hemopoietic stem cells appears to be considerably more difficult in primates than was expected on the basis of the promising results obtained in mice. Nevertheless, the feasibility of gene transfer into bone marrow-derived hemopoietic stem cells and progenitor cells has now been shown in the rhesus monkey with adenoviral vectors ${ }^{89,90}$ and an amphotropic retroviral vector in the common marmoset. ${ }^{91}$ However, although much effort is put in the development of safety-modified lentiviral vectors that are now developed for transduction of human stem cells, safety remains the burning issue with all retroviral vectors. ${ }^{92,93}$ The recently reported occurrence of lymphoma with clonally integrated viral vector in a patient who received gene therapy for severe combined immunedeficiency seems a tragic illustration of the potential risks with integrating viral vectors (http://sciencenow.sciencemag.org; October 3, 2002).

Third, nonhuman primates, in particular the rhesus monkey, provide a useful animal model, in which adenoviral or other viral vector toxicity can be studied. Most risks involved in gene therapy are related to vector systems or the genes they carry. Although viral vectors are genetically modified to minimize their ability to replicate and cause disease, an immune response to viral proteins may still occur and there is a possibility of recombination of the virus resulting in replicationcompetent viruses. The importance of the potential risks of the use of viral vectors is illustrated by the fatal death of a patient in a gene therapy trial. To understand the cause of this tragic event, rhesus monkeys were treated with high dosages of adenoviral vector, similar to the human trial. $^{94}$ From these experiments, evidence emerged that the protein coat of the vector possibly triggered a massive cytokine release by the recipient's immune system.

The use of live attenuated viral vectors has many opportunities and challenges for gene therapy of autoimmune diseases. Perhaps the greatest challenge is to overcome pre-existing immunity to vectors derived from viruses that are widespread in the human population, such as herpes- or adenovirus. Significant advances have been made to attenuate these vectors so that they do not cause disease in a naïve host. However, it remains to be seen if the immunological triggers induced by live vectors can be beneficially applied in an autoimmune setting. Clearly, the tissue-specific delivery and cellspecific expression of the therapeutic transgene are areas of current focus, which will provide us with important advances in the future. Taken together, it can be anticipated that nonhuman primates will increasingly be used to improve delivery systems for gene therapy and to understand and prevent toxicity.

\section{Acknowledgements}

We thank Dr Ronald Bontrop (BPRC), Dr JD Laman (Erasmus Medical Center Rotterdam) for critical reading of the manuscript, Dr Michel Vierboom for editing the manuscript, Mea van de Sman for secretarial assistance and Henk van Westbroek for the artwork. We are grateful to Professor Dr Tom Huizinga (LUMC, Leiden, The Netherlands) for providing Figure 2.

\section{References}

1 Arend WP, Dayer JM. Inhibition of the production and effects of interleukin-1 and tumor necrosis factor alpha in rheumatoid arthritis. Arthritis Rheum 1995; 38: 151-160.

2 Arnason BG. Interferon beta in multiple sclerosis. Clin Immunol Immunopathol 1996; 81: 1-11.

3 Bontrop RE, Otting N, Slierendregt BL, Lanchbury JS. Evolution of major histocompatibility complex polymorphisms and T-cell receptor diversity in primates. Immunol Rev 1995; 143: 33-62.

4 Bontrop RE, Otting N, de Groot NG, Doxiadis GG. Major histocompatibility complex class II polymorphisms in primates. Immunol Rev 1999; 167: 339-350.

5 Toro-Goyco E, Cora EM, Kessler MJ, Martinez-Carrion M. Induction of experimental myasthenia gravis in rhesus monkeys: a model for the study of the human disease. P R Health Sci J 1986; 5: 13-18.

6 Bigazzi P, Rose N. Spontaneous autoimmune thyroiditis in animals as a model of human disease. Prog Allergy 1975; 19: 245274. 
7 Kraan MC et al. Asymptomatic synovitis precedes clinically manifest arthritis. Arthritis Rheum 1998; 41: 1481-1488.

8 Rubin AS et al. Experimental arthropathy induced in rhesus monkeys (Macaca mulatta) by intradermal immunization with native bovine type II collagen. Lab Invest 1987; 57: 524-534.

9 Bakker NP et al. Experimental immune mediated arthritis in rhesus monkeys. A model for human rheumatoid arthritis? Rheumatol Int 1990; 10: 21-29.

10 Terato $\mathrm{K}$ et al. Sex-linked differences in susceptibility of cynomolgus monkeys to type II collagen-induced arthritis. Evidence that epitope-specific immune suppression is involved in the regulation of type II collagen autoantibody formation. Arthritis Rheum 1989; 32: 748-758.

11 Stuart J, Townes A, Ah K. Collagen autoimmune arthritis. Annu Rev Immunol 1984; 2: 199-218.

12 Bakker NP et al. Collagen-induced arthritis in an outbred group of rhesus monkeys comprising responder and nonresponder animals. Relationship between the course of arthritis and collagen-specific immunity. Arthritis Rheum 1991; 34: 616-624.

13 Anthony DD, Haqqi TM. Collagen-induced arthritis in mice: an animal model to study the pathogenesis of rheumatoid arthritis. Clin Exp Rheumatol 1999; 17: 240-244.

14 Staines NA, Wooley PH. Collagen arthritis - what can it teach us? Br J Rheumatol 1994; 33: 798-807.

15 Myers LK, Rosloniec EF, Cremer MA, Kang AH. Collageninduced arthritis, an animal model of autoimmunity. Life Sci 1997; 61: 1861-1878.

16 Bakker NP et al. Resistance to collagen-induced arthritis in a nonhuman primate species maps to the major histocompatibility complex class I region. J Exp Med 1992; 175: 933-937.

17 Slierendregt BL et al. Identification of an Mhc-DPB1 allele involved in susceptibility to experimental autoimmune encephalomyelitis in rhesus macaques. Int Immunol 1995; 7: 16711679.

18 Turner S et al. Identification of antibody epitopes in the CB-11 peptide of bovine type II collagen recognized by sera from arthritis-susceptible and -resistant rhesus monkeys. Clin Exp Immunol 1994; 96: 275-280.

19 t Hart BA, Bakker NP, Jonker M, Bontrop RE. Resistance to collagen-induced arthritis in rats and rhesus monkeys after immunization with attenuated type II collagen. Eur J Immunol 1993; 23: 1588-1594.

20 Noyori $\mathrm{K}$ et al. Binding characteristics of antitype II collagen antibody to the surface of diseased human cartilage as a probe for tissue damage. J Rheumatol 1994; 21: 293-296.

21 t Hart BA et al. Collagen-induced arthritis in rhesus monkeys: evaluation of markers for inflammation and joint degradation. Br J Rheumatol 1998; 37: 314-323.

22 Bakker NP et al. The anti-arthritic and immunosuppressive effects of cyclosporin A on collagen-induced arthritis in the rhesus monkey. Clin Exp Immunol 1993; 93: 318-322.

23 Brok HP et al. Prophylactic and therapeutic effects of a humanized monoclonal antibody against the IL-2 receptor (DACLIZUMAB) on collagen-induced arthritis (CIA) in rhesus monkeys. Clin Exp Immunol 2001; 124: 134-141.

24 van de Loo FA, van den Berg WB. Gene therapy for rheumatoid arthritis. Lessons from animal models, including studies on interleukin-4, interleukin-10, and interleukin-1 receptor antagonist as potential disease modulators. Rheum Dis Clin North Am 2002; 28: 127-149.

25 Vervoordeldonk MJ, Tak PP. Cytokines in rheumatoid arthritis. Curr Rheumatol Rep 2002; 4: 208-217.

26 Apparailly F, Gay S, Jorgensen C. Designing novel therapeutic strategies for rheumatic diseases. Trends Immunol 2001; 22: 537539.

27 Gouze E, Ghivizzani SC, Robbins PD, Evans CH. Gene therapy for rheumatoid arthritis. Curr Rheumatol Rep 2001; 3: 79-85.
28 Muller-Ladner $\mathrm{U}$ et al. Gene transfer of cytokine inhibitors into human synovial fibroblasts in the SCID mouse model. Arthritis Rheum 1999; 42: 490-497.

29 Okamoto $\mathrm{K}$ et al. Induction of apoptosis in the rheumatoid synovium by Fas ligand gene transfer. Gene Therapy 1998; 5: 331 338.

30 Triantaphyllopoulos KA, Williams RO, Tailor H, Chernajovsky Y. Amelioration of collagen-induced arthritis and suppression of interferon-gamma, interleukin-12, and tumor necrosis factor alpha production by interferon-beta gene therapy. Arthritis Rheum 1999; 42: 90-99.

31 Chernajovsky $\mathrm{Y}$ et al. Pathogenic lymphoid cells engineered to express TGF beta 1 ameliorate disease in a collagen-induced arthritis model. Gene Therapy 1997; 4: 553-559.

$32 \mathrm{Kim} \mathrm{SH}$ et al. Effective treatment of established murine collageninduced arthritis by systemic administration of dendritic cells genetically modified to express IL-4. J Immunol 2001; 166: 3499 3505.

33 Lozier JN et al. Toxicity of a first-generation adenoviral vector in rhesus macaques. Hum Gene Ther 2002; 13: 113-124.

34 Apparailly $\mathrm{F}$ et al. Tetracycline-inducible interleukin-10 gene transfer mediated by an adeno-associated virus: application to experimental arthritis. Hum Gene Ther 2002; 13: 1179-1188.

35 Pan RY et al. Therapy and prevention of arthritis by recombinant adeno-associated virus vector with delivery of interleukin-1 receptor antagonist. Arthritis Rheum 2000; 43: 289-297.

36 Conrad CK et al. Safety of single-dose administration of an adeno-associated virus (AAV)- CFTR vector in the primate lung. Gene Therapy 1996; 3: 658-668.

37 Muller-Ladner U et al. Human IL-1Ra gene transfer into human synovial fibroblasts is chondroprotective. J Immunol 1997; 158: 3492-3498.

38 Le CH, Nicolson AG, Morales A, Sewell KL. Suppression of collagen-induced arthritis through adenovirus-mediated transfer of a modified tumor necrosis factor alpha receptor gene. Arthritis Rheum 1997; 40: 1662-1669.

39 Ghivizzani SC et al. Direct adenovirus-mediated gene transfer of interleukin 1 and tumor necrosis factor alpha soluble receptors to rabbit knees with experimental arthritis has local and distal anti-arthritic effects. Proc Natl Acad Sci USA 1998; 95: 4613-4618.

40 Tak PP et al. Inhibitor of nuclear factor kappaB kinase beta is a key regulator of synovial inflammation. Arthritis Rheum 2001; 44: 1897-1907.

41 Boyle DL et al. Intra-articular IL-4 gene therapy in arthritis: antiinflammatory effect and enhanced th2activity. Gene Therapy 1999; 6: 1911-1918.

42 Woods JM et al. Interleukin-13 gene therapy reduces inflammation, vascularization, and bony destruction in rat adjuvantinduced arthritis. Hum Gene Ther 2002; 13: 381-393.

43 Zhang $\mathrm{H}$ et al. Amelioration of collagen-induced arthritis by CD95 (Apo-1/Fas)-ligand gene transfer. J Clin Invest 1997; 100: 1951-1957.

44 Goossens $\mathrm{PH}$ et al. Feasibility of adenovirus-mediated nonsurgical synovectomy in collagen-induced arthritis-affected rhesus monkeys. Hum Gene Ther 1999; 10: 1139-1149.

$45 \mathrm{Kim} \mathrm{JM}$ et al. Angiostatin gene transfer as an effective treatment strategy in murine collagen-induced arthritis. Arthritis Rheum 2002; 46: 793-801.

46 Yin $\mathrm{G}$ et al. Endostatin gene transfer inhibits joint angiogenesis and pannus formation in inflammatory arthritis. Mol Ther 2002; 5: 547-554.

47 Evans $\mathrm{CH}$, Ghivizzani SC, Oligino TJ, Robbins PD. Gene therapy for autoimmune disorders. J Clin Immunol 2000; 20: 334-346.

48 Evans $\mathrm{CH}$ et al. Clinical trial to assess the safety, feasibility, and efficacy of transferring a potentially anti-arthritic cytokine gene to human joints with rheumatoid arthritis. Hum Gene Ther 1996; 7: 1261-1280. 
49 Goossens PH et al. The effect of promoter strength in adenoviral vectors in hyperplastic synovium. Clin Exp Rheumatol 2000; 18: 547-552.

50 Lucchinetti $\mathrm{C}$ et al. Heterogeneity of multiple sclerosis lesions: implications for the pathogenesis of demyelination. Ann Neurol 2000; 47: 707-717.

51 Brok HP et al. Non-human primate models of multiple sclerosis. Immunol Rev 2001; 183: 173-185.

52 Genain CP, Hauser SL. Experimental allergic encephalomyelitis in the New World monkey Callithrix jacchus. Immunol Rev 2001; 183: 159-172.

$53 \mathrm{t}$ Hart BA et al. A new primate model for multiple sclerosis in the common marmoset. Immunol Today 2000; 21: 290-297.

$54 \mathrm{t}$ Hart BA et al. Histopathological characterization of magnetic resonance imaging-detectable brain white matter lesions in a primate model of multiple sclerosis: a correlative study in the experimental autoimmune encephalomyelitis model in common marmosets (Callithrix jacchus). Am J Pathol 1998; 153: 649-663.

55 Antunes SG et al. The common marmoset: a new world primate species with limited Mhc class II variability. Proc Natl Acad Sci USA 1998; 95: 11745-11750.

56 Brok HP et al. Myelin/oligodendrocyte glycoprotein-induced autoimmune encephalomyelitis in common marmosets: the encephalitogenic T cell epitope pMOG24-36 is presented by a monomorphic MHC class II molecule. J Immunol 2000; 165: 10931101.

57 Genain CP et al. Prevention of autoimmune demyelination in non-human primates by a CAMP- specific phosphodiesterase inhibitor. Proc Natl Acad Sci USA 1995; 92: 3601-3605.

58 Brehm U, Piddlesden SJ, Gardinier MV, Linington C. Epitope specificity of demyelinating monoclonal autoantibodies directed against the human myelin oligodendrocyte glycoprotein (MOG). J Neuroimmunol 1999; 97: 9-15.

59 Genain $\mathrm{CP}$ et al. Antibody facilitation of multiple sclerosislike lesions in a nonhuman primate. J Clin Invest 1995; 96: 2966-2974.

60 McFarland HI et al. Determinant spreading associated with demyelination in a nonhuman primate model of multiple sclerosis. J Immunol 1999; 162: 2384-2390.

61 Blezer ELA, Brok HPM, Nicolay K, t Hart BA. Quantifying the progressive inflammatory demyelination of brain white matter in MOG-immunized common marmosets with magnetic resonance imaging. 2002, submitted.

62 Laman JD et al. Protection of marmoset monkeys against EAE by treatment with a murine antibody blocking CD40 (mu5D12). Eur I Immunol 2002; 32: 2218-2228.

63 Boon L et al. Prevention of experimental autoimmune encephalomyelitis in the common marmoset (Callithrix jacchus) using a chimeric antagonist monoclonal antibody against human CD40 is associated with altered B cell responses. J Immunol 2001; 167: 2942-2949.

64 Constantinescu CS et al. IL-12 reverses the suppressive effect of the CD40 ligand blockade on experimental autoimmune encephalomyelitis (EAE). J Neurol Sci 1999; 171: 60-64.

65 Chang JT, Segal BM, Shevach EM. Role of costimulation in the induction of the IL-12/IL-12 receptor pathway and the development of autoimmunity. J Immunol 2000; 164: 100-106.

66 Laman JD et al. Expression of accessory molecules and cytokines in acute EAE in marmoset monkeys (Callithrix jacchus). I Neuroimmunol 1998; 86: 30-45.

67 Brok HP et al. Prevention of experimental autoimmune enchephalomyelitis in common marmosets using a human anti-human IL-12 mAb. J Immunol 2002; 169: 6554-6563.

68 Brok HPM et al. Immunization with MOG peptide 34-56, an immunodominant epitope in multiple sclerosis, evokes a heterogenous pattern of encephalomyelitus in an outbred group of rhesus monkeys (Macaca mulatta). 2002, submitted.
69 Jonker $\mathrm{M}$ et al. Autoimmunity in non-human primates: the role of major histocompatibility complex and T cells, and implications for therapy. Hum Immunol 1991; 32: 31-40.

70 Meinl E et al. Encephalitogenic potential of myelin basic proteinspecific $\mathrm{T}$ cells isolated from normal rhesus macaques. Am J Pathol 1997; 150: 445-453.

$71 \mathrm{t}$ Hart BA, Brok HP, Amor S, Bontrop RE. The major histocompatibility complex influences the ethiopathogenesis of MS-like disease in primates at multiple levels. Hum Immunol 2001; 62: 1371-1381.

72 de Vos AF et al. Redistribution of myelin antigens from demyelinating brain lesions to antigen presenting cells within cervical lymph nodes in monkeys with experimental autoimmune encephalomyelitis. J Immunol 2002; 169: 5415-5423.

73 Lo EH, Singhal AB, Torchilin VP, Abbott NJ. Drug delivery to damaged brain. Brain Res Brain Res Rev 2001; 38: 140-148.

74 Dal Canto RA et al. Local delivery of cytokines by retrovirally transduced antigen-specific TCR + hybridoma cells in experimental autoimmune encephalomyelitis. Eur Cytokine Networks 1998; 9: 83-91.

75 Shaw MK et al. Local delivery of interleukin 4 by retrovirustransduced $\mathrm{T}$ lymphocytes ameliorates experimental autoimmune encephalomyelitis. J Exp Med 1997; 185: 1711-1714.

76 Chen LZ et al. Gene therapy in allergic encephalomyelitis using myelin basic protein- specific T cells engineered to express latent transforming growth factor-beta1. Proc Natl Acad Sci USA 1998; 95: 12516-12521.

77 Costa GL et al. Adoptive immunotherapy of experimental autoimmune encephalomyelitis via T cell delivery of the IL-12 p40 subunit. J Immunol 2001; 167: 2379-2387.

78 Flugel A et al. Anti-inflammatory activity of nerve growth factor in experimental autoimmune encephalomyelitis: inhibition of monocyte transendothelial migration. Eur J Immunol 2001; 31: $11-22$.

79 Willenborg DO, Fordham SA, Cowden WB, Ramshaw IA. Cytokines and murine autoimmune encephalomyelitis: inhibition or enhancement of disease with antibodies to select cytokines, or by delivery of exogenous cytokines using a recombinant vaccinia virus system. Scand I Immunol 1995; 41: 31-41.

80 Piccirillo CA, Prud'homme GJ. Prevention of experimental allergic encephalomyelitis by intramuscular gene transfer with cytokine-encoding plasmid vectors. Hum Gene Ther 1999; 10: 1915-1922

81 Croxford JL et al. Cytokine gene therapy in experimental allergic encephalomyelitis by injection of plasmid DNA-cationic liposome complex into the central nervous system. J Immunol 1998; 160: 5181-5187.

82 Croxford JL, Feldmann M, Chernajovsky Y, Baker D. Different therapeutic outcomes in experimental allergic encephalomyelitis dependent upon the mode of delivery of IL-10: a comparison of the effects of protein, adenoviral or retroviral IL-10 delivery into the central nervous system. J Immunol 2001; 166: 4124-4130.

83 Furlan $\mathrm{R}$ et al. Central nervous system delivery of interleukin 4 by a nonreplicative herpes simplex type 1 viral vector ameliorates autoimmune demyelination. Hum Gene Ther 1998; 9: 2605-2617.

84 Furlan $\mathrm{R}$ et al. Intrathecal delivery of IFN-gamma protects C57BL/6 mice from chronic-progressive experimental autoimmune encephalomyelitis by increasing apoptosis of central nervous system-infiltrating lymphocytes. J Immunol 2001; 167: 1821-1829.

85 Poliani PL et al. Delivery to the central nervous system of a nonreplicative herpes simplex type 1 vector engineered with the interleukin 4 gene protects rhesus monkeys from hyperacute autoimmune encephalomyelitis. Hum Gene Ther 2001; 12: 905920. 
86 Howard MK et al. High efficiency gene transfer to the central nervous system of rodents and primates using herpes virus vectors lacking functional ICP27 and ICP34.5. Gene Therapy 1998; 5: 1137-1147.

87 Nakajima A et al. Antigen-specific T cell-mediated gene therapy in collagen-induced arthritis. J Clin Invest 2001; 107: 1293-1301.

88 Kramer $\mathrm{R}$ et al. Gene transfer through the blood-nerve barrier: NGF-engineered neuritogenic T lymphocytes attenuate experimental autoimmune neuritis. Nat Med 1995; 1: 1162-1166.

89 Dunbar CE. The use of nonhuman primate models to improve gene transfer into haematopoietic stem cells. J Intern Med 2001; 249: 329-338.
90 Van Beusechem VW, Valerio D. Gene transfer into hematopoietic stem cells of nonhuman primates. Hum Gene Ther 1996; 7: 16491668.

91 Hibino $\mathrm{H}$ et al. The common marmoset as a target preclinical primate model for cytokine and gene therapy studies. Blood 1999; 93: 2839-2848.

92 Trono D. Lentiviral vectors: turning a deadly foe into a therapeutic agent. Gene Therapy 2000; 7: 20-23.

93 Dunbar C. Lentivirus get specific. Blood 2002; 99: 397.

94 Stephenson J. Studies illuminate cause of fatal reaction in genetherapy trial. JAMA 2001; 285: 2570. 